\title{
One-way coherent imaging through a distorting medium using four-wave mixing
}

\author{
Amnon Yariv and Thomas L. Koch \\ California Institute of Technology, Pasadena, California 91125
}

Received November 23, 1981

A proposal and an analysis are presented for imaging through a distorting medium using field multiplication by four-wave mixing.

The recent flourish of activity in phase-conjugation optics has revived the interest in real-time imaging through distorting media. Some demonstrations of imaging using thin conventional holograms have been reported earlier ${ }^{1-3}$ as well as real-time imaging schemes using the basic configuration of phase-conjugation optics. $^{4-6}$ In this latter case, the image was obtained on the same side of the distortion as the object, which is a disadvantage, if not an impossibility, in many cases of practical interest. An extension of these imaging ideas to the case of real-time nonlinear mixing media has also been considered?

The scheme proposed here is illustrated in Fig. 1. An image $f\left(\mathbf{x}^{\prime \prime}\right)$ is to be transmitted from plane (3) to plane (1) through an intervening distorting medium that is characterized by a transmittance $\exp \left[-i \Phi\left(\mathbf{x}^{\prime}\right)\right]$. The proposed method of compensation consists of intersecting in a nonlinear medium (NLM) three waves:

(A) A wave $A_{1}$ produced by passing a plane wave through the distortion and then imaging the latter on the NLM.

(B) A picture-bearing wave $A_{2}$ arriving at the NLM from the direction opposite to that of $A_{1}$.

(C) A spherical reference wave $A_{3}$ counterpropagating along the same axis as the picture-bearing wave $A_{2}$.

While we assume that all three waves propagate along the same axis, there will always be some tolerance for wave $A_{1}$ to be slightly off-axis. Our analysis of this situation adopts the following plan: (a) We propagate each of the three fields from their respective starting planes to the NLM. (b) We obtain the nonlinear polarization $P_{\mathrm{NL}}$ in the NLM that is proportional to the product of the three fields. (c) We solve for the field at plane (1) that is radiated by $P_{\mathrm{NL}}$. We will show that this field is of the same form as that which would have been produced at plane (1) by the propagating picture-bearing wave without any distortion.

Using the Fresnel-Kirchhoff diffraction integral and dropping constant phase factors and the time factor $\exp (i \omega t)$ throughout, we write the fields (B) and (C) inside the NLM as:
Picture-wave (B):

$$
\begin{aligned}
E_{2}(\mathbf{x}, z)= & \frac{A_{2}}{\lambda\left(l_{2}-z\right)} e^{i k z} \int \mathrm{d}^{2} x^{\prime \prime} f\left(\mathbf{x}^{\prime \prime}\right) \\
& \times \exp \left[-\frac{i k}{2\left(l_{2}-z\right)}\left|\mathbf{x}-\mathbf{x}^{\prime \prime}\right|^{2}\right] .
\end{aligned}
$$

Reference-wave (C):

$$
E_{3}(\mathbf{x}, z)=A_{3}(z) \exp \left[-i k z-\frac{i k}{2\left(d_{2}+z\right)}|\mathbf{x}|^{2}\right] \text {. }
$$
by

Wave $(A)$ is given in plane $\mathbf{s}$, just to the left of the lens,

$$
E_{1}(\mathbf{s})=\frac{A_{1}}{\lambda d_{1}} \int \mathrm{d}^{2} x^{\prime} \exp \left[-\Phi\left(\mathbf{x}^{\prime}\right)-\frac{i k}{2 d_{1}}\left|\mathbf{x}^{\prime}-\mathbf{s}\right|^{2}\right] .
$$

The effect of the lens is to multiply $E_{1}(\mathbf{s})$ by $\exp \left(i k|\mathbf{s}|^{2} / 2 f\right)$. Next we propagate it to the point ( $\mathbf{x}$, $z$ ) in the NLM, where it becomes

$$
\begin{aligned}
E_{1}(\mathbf{x}, z)= & \frac{A_{1}}{\lambda^{2} d_{1}\left(l_{1}+z\right)} e^{i k z} \int \mathrm{d}^{2} x^{\prime} \int \mathrm{d}^{2} s \\
& \times \exp \left[-i \Phi\left(\mathbf{x}^{\prime}\right)-\frac{i k}{2 d_{1}}\left|\mathbf{x}^{\prime}-\mathbf{s}\right|^{2}\right] \\
& \times \exp \left[\frac{i k}{2 f}|\mathbf{s}|^{2}-\frac{i k}{2\left(l_{1}+z\right)}|\mathbf{x}-\mathbf{s}|^{2}\right] .
\end{aligned}
$$

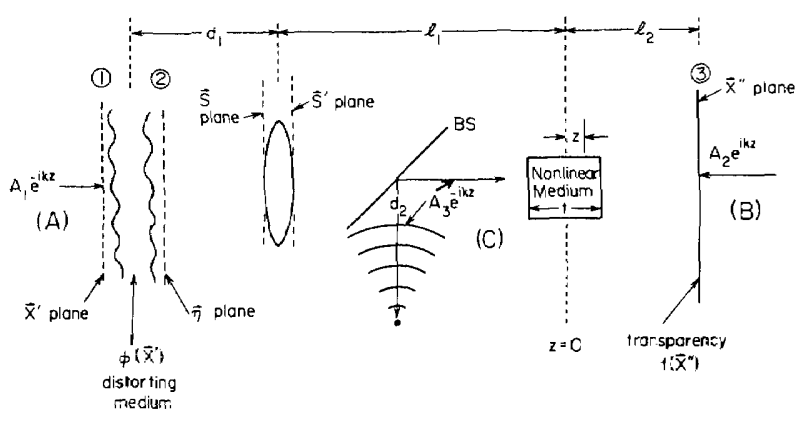

Fig. 1. The configuration for propagating an image from plane (3) through a distorting medium to plane (1) with no distortion. 
If the NLM is short, i.e., $z \ll d_{2}, l_{1}, l_{2}$, in the phase factors, we can expand

$$
\begin{aligned}
& \frac{1}{d_{2}+z} \approx \frac{1}{d_{2}}-\frac{z}{d_{2}{ }^{2}}, \frac{1}{l_{1}+z} \approx \frac{1}{l_{1}}- \frac{z}{l_{1}{ }^{2}}, \\
& \frac{1}{l_{2}-z} \approx \frac{1}{l_{2}}+\frac{z}{l_{2}{ }^{2}}
\end{aligned}
$$

and ignore the $z$ dependence of the denominators preceding the integrals. These relations will be used in what follows.

The intersection of the three waves in the NLM gives rise to a polarization that is proportional to the product of all three. The term of interest is that involving $E_{1}{ }^{*} E_{2} E_{3}$ since it oscillates at the fundamental frequency $\omega$ and the term $E_{1}{ }^{*}$ signifies a reversal of the sign of the distortion. Also this polarization radiates in the desired $-z$ direction toward plane (1). Thus the nonlinear polarization induced in the NLM is given by

$$
P_{\mathrm{NL}}=\chi^{(3)} E_{1} * E_{2} E_{3},
$$

where $\chi^{(3)}$ is the third-order nonlinear susceptibility of the medium. Using the expressions (1), (2), and (4) for the fields as well as (6) and assuming that the distortion is imaged by the lens at $z=0$ so that $f^{-1}=d_{1}^{-1}+l_{1}^{-1}$, we obtain

$$
\begin{aligned}
P_{\mathrm{NL}}(\mathbf{x}, z)= & \frac{\chi^{(3)} A_{1} * A_{2} A_{3}(z=0)}{\lambda^{3} d_{1} l_{1} l_{2}} e^{i k z} \\
& \times \iiint \mathrm{d}^{2} x^{\prime \prime} \mathrm{d}^{2} x^{\prime} \mathrm{d}^{2} s f\left(\mathbf{x}^{\prime \prime}\right) e^{i \Phi\left(\mathbf{x}^{\prime}\right)} \\
& \times \exp \left(-\frac{i k}{2 d_{2}}|\mathbf{x}|^{2}-\frac{i k z}{2 d_{2}{ }^{2}}|\mathbf{x}|^{2}\right) \\
& \times \exp \left(-\frac{i k}{2 l_{2}}\left|\mathbf{x}-\mathbf{x}^{\prime \prime}\right|^{2}-\frac{i k z}{2 l_{2}{ }^{2}}\left|\mathbf{x}-\mathbf{x}^{\prime \prime}\right|^{2}\right) \\
& \times \exp \left[\frac{i k}{2 d_{1}}\left|\mathbf{x}^{\prime}\right|^{2}+\frac{i k}{2 l_{1}}|\mathbf{x}|^{2}\right. \\
& \left.-\frac{i k}{d_{1}}\left(\mathbf{x}^{\prime}+\frac{d_{1}}{l_{1}} \mathbf{x}\right) \cdot \mathbf{s}-\frac{i k z}{2 l_{1}{ }^{2}}|\mathbf{x}-\mathbf{s}|^{2}\right] .
\end{aligned}
$$

The field at the $s^{\prime}$ plane that is due to $P_{\mathrm{NL}}$ is obtained using a Green function solution

$$
E\left(\mathbf{s}^{\prime}\right)=\frac{4 \pi^{2}}{\lambda^{2}} \int_{v} \frac{P_{\mathrm{NL}}(\mathbf{x}, z) e^{-i k r}}{r} \mathrm{~d}^{2} x \mathrm{~d} z,
$$

where $r=\left[\left|\mathbf{x}-\mathbf{s}^{\prime}\right|^{2}+\left(l_{1}+z\right)^{2}\right]^{1 / 2}$. In the Fresnel approximation limit this becomes

$$
\begin{aligned}
& E\left(\mathbf{s}^{\prime}\right)=\frac{4 \pi^{2}}{\lambda^{2} l_{1}} \int_{v} \mathrm{~d}^{2} x \mathrm{~d} z P_{\mathrm{NL}}(\mathbf{x}, \mathrm{z}) \\
& \quad \times \exp \left(-i k z-\frac{i k}{2 l_{1}}\left|\mathbf{x}-\mathbf{s}^{\prime}\right|^{2}+\frac{i k z}{2 l_{1}{ }^{2}}\left|\mathbf{x}-\mathbf{s}^{\prime}\right|^{2}\right) .
\end{aligned}
$$

The effect of the lens again is to multiply $E\left(\mathbf{s}^{\prime}\right)$ by $\exp \left(i k\left|\mathbf{s}^{\prime}\right|{ }^{2} / 2 f\right)$. The resulting field propagates to the $\eta$ plane (2) and is there multiplied by the distortion function $\exp [-i \Phi(\eta)]$. If we recall the imaging condition $f^{-1}=d_{1}^{-1}+l_{1}^{-1}$ we obtain after some algebra the desired output field

$$
\begin{aligned}
E(\eta)= & \frac{4 \pi^{2} \chi^{(3)} A_{1} * A_{2} A_{3}}{\lambda^{6} d_{1}{ }^{2} l_{1}{ }^{2} l_{2}} e^{-i \Phi(\eta)} \\
& \times \iiint \iiint \mathrm{d} z \mathrm{~d}^{2} x \mathrm{~d}^{2} x^{\prime} \mathrm{d}^{2} x^{\prime \prime} \mathrm{d}^{2} s \mathrm{~d}^{2} s^{\prime} \\
& \times e^{i \Phi\left(\mathbf{x}^{\prime}\right)} f\left(\mathbf{x}^{\prime \prime}\right) \exp \left(-\frac{i k}{2 d_{1}}|\eta|^{2}-\frac{i k}{2 d_{2}}|\mathbf{x}|^{2}\right. \\
& \left.+\frac{i k}{2 d_{1}}\left|\mathbf{x}^{\prime}\right|^{2}-\frac{i k}{2 l_{2}}\left|\mathbf{x}-\mathbf{x}^{\prime \prime}\right|^{2}\right) \\
& \times \exp \left[-\frac{i k}{d_{1}}\left(\mathbf{x}^{\prime}+\frac{d_{1}}{l_{1}} \mathbf{x}\right) \cdot \mathbf{s}\right] \\
& \times \exp \left[\frac{i k}{d_{1}}\left(\eta+\frac{d_{1}}{l_{1}} \mathbf{x}\right) \cdot \mathbf{s}^{\prime}\right] \\
& \times\left\{\operatorname { e x p } \left(\frac{i k z}{2 d_{2}{ }^{2}}|\mathbf{x}|^{2}-\frac{i k z}{2 l_{2}{ }^{2}}\left|\mathbf{x}-\mathbf{x}^{\prime \prime}\right|^{2}\right.\right. \\
& \left.\left.+\frac{i k z}{2 l_{1}{ }^{2}}\left|\mathbf{x}-\mathbf{s}^{\prime}\right|^{2}-\frac{i k z}{2 l_{1}{ }^{2}}|\mathbf{x}-\mathbf{s}|^{2}\right)\right\}
\end{aligned}
$$

For sufficiently thin NLM such that

$$
k z \frac{\mid \mathbf{x} \text { or } \mathbf{x}^{\prime \prime} \text { or } \mathbf{s} \text { or }\left.\mathbf{s}^{\prime}\right|^{2}}{\left(l_{1} \text { or } l_{2} \text { or } d_{2}\right)^{2}} \ll \pi
$$

the last factor in the curly brackets is very nearly unity. Ignoring finite aperature effects, the integrations over $\mathbf{s}$ and $\mathbf{s}^{\prime}$ yield

$$
\left(d_{1} \lambda\right)^{2} \delta\left(\mathbf{x}^{\prime}+\frac{d_{1}}{l_{1}} \mathbf{x}\right) \text { and }\left(l_{1} \lambda\right)^{2} \delta\left(\mathbf{x}+\frac{l_{1}}{d_{1}} \eta\right),
$$

respectively. These collapse the $\mathbf{x}^{\prime}$ and $\mathbf{x}$ integrations to get $\mathbf{x}^{\prime} \rightarrow \eta, \mathbf{x} \rightarrow-\left(l_{1} / d_{1}\right) \eta$, and then Eq. (10) becomes

$$
\begin{aligned}
E(\eta)= & {\left[4 \pi^{2} \chi^{(3)} A_{1}{ }^{*} A_{3} t / \lambda\right] \exp \left(-\frac{i k l_{1}{ }^{2}}{2 d_{2} d_{1}^{2}}|\eta|^{2}\right) } \\
& \times \frac{A_{2}}{\lambda l_{2}} \int \mathrm{d}^{2} x^{\prime \prime} f\left(\mathbf{x}^{\prime \prime}\right) \exp \left(-\frac{i k}{2 l_{2}}\left|\frac{l_{1}}{d_{1}} \eta+\mathbf{x}^{\prime \prime}\right|^{2}\right),
\end{aligned}
$$

where $t$ is the thickness (along $z$ ) of the NLM.

If $d_{2} \rightarrow \infty$, i.e., the reference wave (C) is a plane wave, then we have according to Eq. (11) in the $\eta$ plane an enlarged (by $d_{1} / l_{1}$ ) and inverted version of the original object field having propagated from an effective plane a distance $l_{2}$ to the right without distortion. For finite $d_{2}$ the resulting field is the same as in Eq. (11) with the additional effect of a lens with focal length $d_{2} d_{1}^{2} / l_{1}^{2}$ in the $\eta$ plane. This completes the proof.

The factor in the square brackets preceding the integral in Eq. (11) represents the change in the amplitude of the image field between planes (3) and (1) that is due to nonlinear mixing. This factor is therefore the effective amplitude gain

$$
G \approx 4 \pi^{2} \chi^{(3)} A_{1}{ }^{*} A_{3} t / \lambda
$$

and can exceed unity for sufficiently strong fields. The proposed scheme can thus be used for image amplification as well as correction.

In conclusion, a method for a one-way imaging through a turbulent medium using four-wave mixing in a nonlinear medium has been proposed and analyzed. 
The authors would like to acknowledge especially insightful discussions with and valuable suggestions of B. Fischer, J. White, and M. Cronin-Golomb.

This work was supported by the U.S. Army Research Office, Durham, North Carolina.

\section{References}

1. E. N. Leith and J. Upatnieks, "Holographic imagery through diffusing media," J. Opt. Soc. Am. 56, 523 (1966).

2. H. Kogelnik, "Holographic image projection through inhomogenious media," Bell Syst. Tech. J. 44, 2451 (1965).
3. J. W. Goodman et al., "Wavefront reconstruction imaging through random media," Appl. Phys. Lett. 8, 311 (1966).

4. B. I. Stepanov, E. V. Ivakin, and A. S. Rubanov, "On registration of plane wave and volume dynamic holograms in bleachable substances," Dokl. Akad. Nauk SSSR 196, 567 (1971).

5. J. P. Woerdman, "Some optical and electrical properties of a laser generated free carrier plasma in Si," Ph.D. Thesis, U. of Amsterdam (Dec. 1971). Also republished in Philips Res. Dept. Suppl. 1971, No. 7.

6. A. Yariv, "On transmission and recovery of three dimensional image information in optical waveguides," J. Opt. Soc. Am. 66, 301 (1976).

7. T. R. O'Meara, "Applications of nonlinear phase conjugation in compensated active media," Proc. Soc. PhotoOpt. Instrum. Eng. 168, 542 (1979). 